\title{
Decoherence properties of arbitrarily long histories*
}

\author{
Artur Scherer ${ }^{\dagger}$ and Andrei N. Soklakov ${ }^{\ddagger}$ \\ Department of Mathematics, Royal Holloway, \\ University of London, Egham, Surrey, TW20 OEX, UK.
}

(Dated: 15 Sept, 2004)

\begin{abstract}
Within the decoherent histories formulation of quantum mechanics, we consider arbitrarily long histories constructed from a fixed projective partition of a finite-dimensional Hilbert space. We review some of the decoherence properties of such histories including simple necessary decoherence conditions and the dependence of decoherence on the initial state. Here we make a first step towards generalization of our earlier results [Scherer and Soklakov, e-print: quant-ph/0405080, (2004) and Scherer et al., Phys. Lett. A 326, 307, (2004)] to the case of approximate decoherence.
\end{abstract}

PACS numbers: 03.65.Ca, 03.65.Ta, 03.65.Yz, 05.70.Ln

Keywords: decoherent histories

\footnotetext{
* in Proceedings of the 7th International Conference on Quantum Communication, Measurement and Computing (QCMC'04), edited by Stephen M. Barnett (AIP Press, Melville, NY, 2004).

$\dagger$ the corresponding author: artur.scherer@uni-konstanz.de; a.scherer@rhul.ac.uk

† a.soklakov@rhul.ac.uk
} 


\section{INTRODUCTION}

The formalism of decoherent histories was introduced to provide a self-contained description of closed quantum systems that does not rely on either the external observer nor on the existence of classical devices [3, 4, 5, 6, 7]. It has been successfully applied in various fields of

quantum theory including quantum cosmology [8], derivation of effective classical dynamics from the fundamental quantum dynamical laws [6, 7, 9], and the study of the coarse-grained evolution of iterated quantum maps [10]. Recently the formalism of decoherent histories has also been applied for investigating classicality in quantum information processing [11].

The decoherent histories formalism predicts probabilities for quantum histories, i.e. ordered sequences of quantum-mechanical "propositions". Mathematically, these propositions are represented by projectors. In particular, an exhaustive set of mutually exclusive propositions corresponds to a complete set of mutually orthogonal projectors. Due to quantum interference, however, one cannot always assign probabilities to a set of histories in a consistent way. For this to be possible, the set of histories must be decoherent. Decoherence of histories ensures that the assigned probabilities obey the standard probability sum rules.

In Refs. [1, 2] a special, but very important class of histories was introduced, namely, the class of histories that are constructed from a fixed projective partition of a finite-dimensional Hilbert space. In the context of exact decoherence a number of results were obtained regarding decoherence properties of such histories. In particular, simple necessary decoherence conditions were derived and the dependence of decoherence on the initial state was investigated. In this paper we give a brief review of these results and make a first step towards generalizations of these results to the case of approximate decoherence.

The paper is organized as follows. After introducing our framework we first review the results obtained in 1, 2] for the case of exact decoherence. In the second part of the paper approximate decoherence of histories is introduced and the corresponding implications examined.

\section{OUR SETTING}

Definition 1: A set of projectors $\left\{P_{\mu}\right\}$ on a Hilbert space $\mathcal{H}$ is called a projective partition of $\mathcal{H}$, if $\forall \mu, \mu^{\prime}: P_{\mu} P_{\mu^{\prime}}=\delta_{\mu \mu^{\prime}} P_{\mu}$ and $\sum_{\mu} P_{\mu}=\mathbb{1}_{\mathcal{H}}$. Here, $\mathbb{1}_{\mathcal{H}}$ denotes the unit operator. 
We call a projective partition fine-grained if all projectors are one-dimensional, i.e., if $\forall \mu$ $\operatorname{dim}\left(\operatorname{supp}\left(P_{\mu}\right)\right)=1$, and coarse-grained otherwise.

Definition 2: Given a projective partition $\left\{P_{\mu}\right\}$ of a Hilbert space $\mathcal{H}$, let us denote by $\mathcal{K}\left[\left\{P_{\mu}\right\} ; k\right]:=\left\{h_{\vec{\alpha}}: h_{\vec{\alpha}}=\left(P_{\alpha_{1}}, P_{\alpha_{2}}, \ldots, P_{\alpha_{k}}\right) \in\left\{P_{\mu}\right\}^{k}\right\}$ the corresponding exhaustive set of mutually exclusive histories of length $k$. Histories are thus defined to be ordered sequences of projection operators, corresponding to quantum-mechanical propositions. Note that we restrict ourselves to histories constructed from a fixed projective partition: the projectors $P_{\alpha_{j}}$ within the sequences are all chosen from the same partition for all "times" $j=1, \ldots, k$. Definition 3: Given a Hilbert space $\mathcal{H}$ and a projective partition $\left\{P_{\mu}\right\}$ of $\mathcal{H}$, we denote by $\mathcal{S}$ the set of all density operators on $\mathcal{H}$ and by $\mathcal{S}_{\left\{P_{\mu}\right\}}$ the discrete set of "partition states" induced by the partition $\left\{P_{\mu}\right\}$ via normalization: $\mathcal{S}_{\left\{P_{\mu}\right\}}:=\left\{P_{\nu} / \operatorname{Tr}\left[P_{\nu}\right]: P_{\nu} \in\left\{P_{\mu}\right\}\right\}$. Furthermore, a state $\rho \in \mathcal{S}$ is called classical with respect to (w.r.t.) the partition $\left\{P_{\mu}\right\}$ if it is block-diagonal w.r.t. $\left\{P_{\mu}\right\}$, i.e., if $\rho=\sum_{\mu} P_{\mu} \rho P_{\mu}$. The set of all such classical states is denoted by $\mathcal{S}_{\left\{P_{\mu}\right\}}^{c l}$.

An initial state $\rho \in \mathcal{S}$ and a unitary dynamics generated by a unitary map $U: \mathcal{H} \rightarrow \mathcal{H}$ induce a probabilistic structure on the event algebra associated with $\mathcal{K}\left[\left\{P_{\mu}\right\} ; k\right]$, if certain consistency conditions are fulfilled. These are given in terms of properties of the decoherence functional $\mathcal{D}_{U, \rho}[\cdot, \cdot]$ on $\mathcal{K}\left[\left\{P_{\mu}\right\} ; k\right] \times \mathcal{K}\left[\left\{P_{\mu}\right\} ; k\right]$, defined by

$$
\mathcal{D}_{U, \rho}\left[h_{\vec{\alpha}}, h_{\vec{\beta}}\right]:=\operatorname{Tr}\left[C_{\vec{\alpha}} \rho C_{\vec{\beta}}^{\dagger}\right],
$$

where

$$
\begin{aligned}
C_{\vec{\alpha}} & :=\left(U^{\dagger k} P_{\alpha_{k}} U^{k}\right)\left(U^{\dagger k-1} P_{\alpha_{k-1}} U^{k-1}\right) \ldots\left(U^{\dagger} P_{\alpha_{1}} U\right) \\
& =U^{\dagger k} P_{\alpha_{k}} U P_{\alpha_{k-1}} U \ldots P_{\alpha_{2}} U P_{\alpha_{1}} U
\end{aligned}
$$

The set $\mathcal{K}\left[\left\{P_{\mu}\right\} ; k\right]$ is said to be decoherent or consistent with respect to a given unitary map $U: \mathcal{H} \rightarrow \mathcal{H}$ and a given initial state $\rho \in \mathcal{S}$, if

$$
\mathcal{D}_{U, \rho}\left[h_{\vec{\alpha}}, h_{\vec{\beta}}\right] \propto \delta_{\vec{\alpha} \vec{\beta}} \equiv \prod_{j=1}^{k} \delta_{\alpha_{j} \beta_{j}}
$$

for all $h_{\vec{\alpha}}, h_{\vec{\beta}} \in \mathcal{K}\left[\left\{P_{\mu}\right\} ; k\right]$. These are the consistency conditions. If they are fulfilled, probabilities may be assigned to the histories and are given by the diagonal elements of 
the decoherence functional, $p\left[h_{\vec{\alpha}}\right]=\mathcal{D}_{U, \rho}\left[h_{\vec{\alpha}}, h_{\vec{\alpha}}\right]$. The conditions given above are known as medium decoherence [7]. It has recently been shown that consideration of the weaker consistency conditions that had been proposed in the literature [3, 4] is problematic [12].

\section{MOTIVATION}

Whether the decoherence condition (3) is fulfilled or not depends on the initial state, the unitary dynamics of the system and the propositions from which the histories are constructed. The dependence on the initial state is connected to one of the central questions of the decoherence programme: the question of how the classical features of our world emerge from the initial quantum state of the Universe.

A rather more technical motivation for the research presented in this paper comes from the need of simpler decoherence conditions. In general, it is very difficult to decide whether a given set of histories is decoherent. With increasing length of the histories checking the decoherence conditions (3) soon becomes extremely cumbersome. This is especially true when the system dynamics is difficult to simulate as, e.g., in the case of chaotic quantum maps, for which typically only the first iteration is known in closed analytical form. Establishing decoherence directly, by computing the off-diagonal elements of the decoherence functional,

would require enormous computational resources in the case of large history lengths. It would therefore be of great practical importance to have a simple decoherence criterion that uses only a single iteration of the unitary map. Sufficient conditions of this type can trivially be found. Our analysis concentrates on necessary single-iteration decoherence conditions.

\section{RESULTS FOR EXACT DECOHERENCE}

In 2] we have proven the following theorem.

Theorem 1: Let a projective partition $\left\{P_{\mu}\right\}$ of a finite dimensional Hilbert space $\mathcal{H}$ and a unitary map $U$ on $\mathcal{H}$ be given. Then the following three statements are equivalent:

$$
\begin{aligned}
& \text { (a) } \forall \rho \in \mathcal{S}_{\left\{P_{\mu}\right\}} \forall k \in \mathbb{N} \forall h_{\vec{\alpha}}, h_{\vec{\beta}} \in \mathcal{K}\left[\left\{P_{\mu}\right\} ; k\right]: \mathcal{D}_{U, \rho}\left[h_{\vec{\alpha}}, h_{\vec{\beta}}\right] \propto \delta_{\vec{\alpha} \vec{\beta}} \\
& \text { (b) } \forall P_{\mu^{\prime}}, P_{\mu^{\prime \prime}} \in\left\{P_{\mu}\right\} \forall n \in \mathbb{N}:\left[U^{n} P_{\mu^{\prime}}\left(U^{\dagger}\right)^{n}, P_{\mu^{\prime \prime}}\right]=0 \\
& \text { (c) } \forall \rho \in \mathcal{S} \forall k \in \mathbb{N} \forall h_{\vec{\alpha}}, h_{\vec{\beta}} \in \mathcal{K}\left[\left\{P_{\mu}\right\} ; k\right]: \mathcal{D}_{U, \rho}\left[h_{\vec{\alpha}}, h_{\vec{\beta}}\right] \propto \delta_{\vec{\alpha} \vec{\beta}} .
\end{aligned}
$$


The implication $(\mathrm{a}) \Rightarrow(\mathrm{c})$ of the theorem leads to an interesting conclusion concerning the dependence of decoherence on the initial state: the decoherence of histories of arbitrary length for all initial states from the set $\mathcal{S}_{\left\{P_{\mu}\right\}}$ implies decoherence of such histories for arbitrary initial states $\rho \in \mathcal{S}$. Note that the set $\mathcal{S}_{\left\{P_{\mu}\right\}}$ can be viewed as the smallest natural set of states that is associated with our framework. It is discrete and may consist of just two elements (in the case of "yes-no" propositions). The set $\mathcal{S}$, on the other hand, contains the continuum of all possible states.

The above theorem also provides a necessary single-iteration decoherence condition that is applicable to arbitrary coarse-grainings. This generalizes the simple condition derived in an earlier paper [1] for the case of fine-grained histories. In 1] it was shown that, in the case of fine-grained partitions, sets of histories of arbitrary length decohere for all classical initial states $\rho \in \mathcal{S}_{\left\{P_{\mu}\right\}}^{c l}$ only if the unitary dynamics preserves the classicality of states, i.e. only if

$$
\forall \rho \in \mathcal{S}_{\left\{P_{\mu}\right\}}^{c l}: U \rho U^{\dagger} \in \mathcal{S}_{\left\{P_{\mu}\right\}}^{c l} .
$$

Unfortunately, condition (4) fails to be necessary [13] in the coarse-grained case [1]. The above theorem provides a necessary single-iteration condition that now applies to arbitrary coarse-grainings and is equivalent to (4) in the fine-grained case. According to the implication $(\mathrm{a}) \Rightarrow(\mathrm{b})$ of the theorem the medium decoherence condition is satisfied for all classical initial states $\rho \in \mathcal{S}_{\left\{P_{\mu}\right\}}^{c l}$ and arbitrarily long histories, i.e.,

$$
\forall \rho \in \mathcal{S}_{\left\{P_{\mu}\right\}}^{c l} \forall k \in \mathbb{N} \forall h_{\vec{\alpha}}, h_{\vec{\beta}} \in \mathcal{K}\left[\left\{P_{\mu}\right\} ; k\right]: \mathcal{D}_{U, \rho}\left[h_{\vec{\alpha}}, h_{\vec{\beta}}\right] \propto \delta_{\vec{\alpha} \vec{\beta}},
$$

only if the following necessary condition is fulfilled:

$$
\forall P_{\mu^{\prime}}, P_{\mu^{\prime \prime}} \in\left\{P_{\mu}\right\}:\left[U P_{\mu^{\prime}} U^{\dagger}, P_{\mu^{\prime \prime}}\right]=0 .
$$

\section{GENERALISATION TO APPROXIMATE DECOHERENCE}

Condition (3) is the condition for exact decoherence. In most of physical models, however, decoherence of histories can be established only approximately (cf. e.g. [6]). It is therefore desirable to generalise the above results to the case of approximate decoherence. Let us first explain what is meant by approximate decoherence. An absolutely consistent assignment of probabilities to a given set of histories requires that whenever we bundle up the given histories to coarser-grained histories then the probability for each such coarser-grained history 
must be equal to the sum of the probabilities for its constituent finer-grained histories, and this has to be true for all possible coarse-grainings. If these probability sum rules are fulfilled only approximately, for all possible coarse-grainings of a given set of finer-grained histories, then we get an approximately consistent assignment of probabilities and call the given set of histories approximately decoherent. Quantitatively, one requires that the probability sum rules are satisfied to some order $\epsilon$, meaning that the interference terms are suppressed by a very small factor $\epsilon \ll 1$ compared to the sums over the probabilities, for all possible coarsegrainings. A condition that proved to be useful for approximate decoherence is (cf. Ref. [6] and [14])

$$
\left|\mathcal{D}_{U, \rho}\left[h_{\vec{\alpha}}, h_{\vec{\beta}}\right]\right|<\epsilon\left(\mathcal{D}_{U, \rho}\left[h_{\vec{\alpha}}, h_{\vec{\alpha}}\right] \mathcal{D}_{U, \rho}\left[h_{\vec{\beta}}, h_{\vec{\beta}}\right]\right)^{\frac{1}{2}} \quad \text { for } h_{\vec{\alpha}} \neq h_{\vec{\beta}}
$$

In 6] it was shown that with this condition most (in a statistical sense) probability sum rules are satisfied to order $\epsilon$ provided the number of all possible histories $h_{\vec{\alpha}}$ is large. Here we assume a stronger condition, which guarantees that all probability sum rules are satisfied to the order $\epsilon$, for all possible coarse-grainings, namely,

$$
\left|\mathcal{D}_{U, \rho}\left[h_{\vec{\alpha}}, h_{\vec{\beta}}\right]\right|<\epsilon \frac{\left(\mathcal{D}_{U, \rho}\left[h_{\vec{\alpha}}, h_{\vec{\alpha}}\right] \mathcal{D}_{U, \rho}\left[h_{\vec{\beta}}, h_{\vec{\beta}}\right]\right)^{\frac{1}{2}}}{\left|\mathcal{K}\left[\left\{P_{\mu}\right\} ; k\right]\right|} \text { for } h_{\vec{\alpha}} \neq h_{\vec{\beta}},
$$

where $\left|\mathcal{K}\left[\left\{P_{\mu}\right\} ; k\right]\right|$ denotes the number of elements in the set $\mathcal{K}\left[\left\{P_{\mu}\right\} ; k\right]$, which is the number of all possible histories $h_{\vec{\alpha}}$. It is bounded from above by $d^{k}$ with $d$ being the dimension of the Hilbert space, $d=\operatorname{dim} \mathcal{H}$.

The only difficult part in the proof of Theorem 1 was to show the implication "(a) $\Rightarrow(\mathrm{b})$ ". As a first step towards proving analogous results for approximate decoherence, we confine ourselves to generalizing just this part of Theorem 1. Instead of exact decoherence we now assume approximate decoherence of histories for arbitrary history lengths $k$ and for all initial states $\rho \in \mathcal{S}_{\left\{P_{\mu}\right\}}$, i.e., we replace in the statement (a) of Theorem 1 the exact decoherence condition (3) by our approximate decoherence condition (8). The task now is to show that the statement (b) of Theorem 1 is still implied in some approximate sense. The derivation of this implication can only be sketched in this paper. It is based on a Lemma on uniform recurrence in finite dimensional Hilbert spaces [1] and is done in a similar way as in the proof of Theorem 1 in Ref. [2]. Using the trivial relation

$$
\left(\mathcal{D}_{U, \rho}\left[h_{\vec{\alpha}}, h_{\vec{\alpha}}\right] \mathcal{D}_{U, \rho}\left[h_{\vec{\beta}}, h_{\vec{\beta}}\right]\right)^{\frac{1}{2}} \leq \frac{1}{2}\left(\mathcal{D}_{U, \rho}\left[h_{\vec{\alpha}}, h_{\vec{\alpha}}\right]+\mathcal{D}_{U, \rho}\left[h_{\vec{\beta}}, h_{\vec{\beta}}\right]\right)
$$


together with the techniques of Ref. 2] we can show that the modified assumption (a) necessarily implies that

$$
\begin{aligned}
& \forall n \in \mathbb{N} \forall \mu_{0}, \mu^{\prime}, \mu^{\prime \prime} \quad \text { with } \quad \mu^{\prime} \neq \mu^{\prime \prime}: \\
& \left|\operatorname{Tr}\left[P_{\mu^{\prime \prime}}\left(U^{n} P_{\mu_{0}} U^{\dagger n}\right) P_{\mu^{\prime}}\left(U^{n} P_{\mu_{0}} U^{\dagger n}\right) P_{\mu^{\prime \prime}}\right]\right|<d \epsilon .
\end{aligned}
$$

This condition is equivalent to

$$
\forall n \in \mathbb{N} \forall \mu_{0}, \mu^{\prime}, \mu^{\prime \prime} \quad \text { with } \quad \mu^{\prime} \neq \mu^{\prime \prime}:\left\|P_{\mu^{\prime}}\left(U^{n} P_{\mu_{0}} U^{\dagger n}\right) P_{\mu^{\prime \prime}}\right\|_{2}<\sqrt{d \epsilon},
$$

where $\|A\|_{2}:=\sqrt{\operatorname{Tr}\left[A^{\dagger} A\right]}$ denotes the Hilbert-Schmidt operator norm for any operator $A$. It then follows, for all $n \in \mathbb{N}$ and for all $\mu_{0}, \mu^{\prime \prime}$, that

$$
\begin{aligned}
\left\|\left[\left(U^{n} P_{\mu_{0}} U^{\dagger n}\right), P_{\mu^{\prime \prime}}\right]\right\|_{2} & =\left\|\left(U^{n} P_{\mu_{0}} U^{\dagger n}\right) P_{\mu^{\prime \prime}}-P_{\mu^{\prime \prime}}\left(U^{n} P_{\mu_{0}} U^{\dagger n}\right)\right\|_{2} \\
& =\left\|\left(\sum_{\mu^{\prime}} P_{\mu^{\prime}}\right)\left(U^{n} P_{\mu_{0}} U^{\dagger n}\right) P_{\mu^{\prime \prime}}-P_{\mu^{\prime \prime}}\left(U^{n} P_{\mu_{0}} U^{\dagger n}\right)\left(\sum_{\mu^{\prime}} P_{\mu^{\prime}}\right)\right\|_{2} \\
& \leq \sum_{\substack{\mu^{\prime} \\
\mu^{\prime} \neq \mu^{\prime \prime}}}\left\|P_{\mu^{\prime}}\left(U^{n} P_{\mu_{0}} U^{\dagger n}\right) P_{\mu^{\prime \prime}}-P_{\mu^{\prime \prime}}\left(U^{n} P_{\mu_{0}} U^{\dagger n}\right) P_{\mu^{\prime}}\right\|_{2} \\
& \leq \sum_{\substack{\mu^{\prime} \\
\mu^{\prime} \neq \mu^{\prime \prime}}}\left\{\left\|P_{\mu^{\prime}}\left(U^{n} P_{\mu_{0}} U^{\dagger n}\right) P_{\mu^{\prime \prime}}\right\|_{2}+\left\|P_{\mu^{\prime \prime}}\left(U^{n} P_{\mu_{0}} U^{\dagger n}\right) P_{\mu^{\prime}}\right\|_{2}\right\} \\
& <2\left(\sharp \mu^{\prime}\right) \sqrt{d \epsilon} \leq 2 d \sqrt{d \epsilon}=2 d^{\frac{3}{2}} \sqrt{\epsilon},
\end{aligned}
$$

i.e. $\left\|\left[\left(U^{n} P_{\mu_{0}} U^{\dagger n}\right), P_{\mu^{\prime \prime}}\right]\right\|_{2}<2 d^{\frac{3}{2}} \sqrt{\epsilon}$ for all $n \in \mathbb{N}$ and for all $\mu_{0}, \mu^{\prime \prime}$. We thus get the following generalisation of the implication $(\mathrm{a}) \Rightarrow(\mathrm{b})$ of Theorem 1 :

Theorem 2: Let a projective partition $\left\{P_{\mu}\right\}$ of a finite dimensional Hilbert space $\mathcal{H}, a$ unitary map $U$ on $\mathcal{H}$, and a small $\epsilon>0$ be given. Then

$\forall \rho \in \mathcal{S}_{\left\{P_{\mu}\right\}} \forall k \in \mathbb{N} \forall h_{\vec{\alpha}}, h_{\vec{\beta}} \in \mathcal{K}\left[\left\{P_{\mu}\right\} ; k\right]$ with $h_{\vec{\alpha}} \neq h_{\vec{\beta}}:$

$$
\left|\mathcal{D}_{U, \rho}\left[h_{\vec{\alpha}}, h_{\vec{\beta}}\right]\right|<\epsilon \frac{\left(\mathcal{D}_{U, \rho}\left[h_{\vec{\alpha}}, h_{\vec{\alpha}}\right] \mathcal{D}_{U, \rho}\left[h_{\vec{\beta}}, h_{\vec{\beta}}\right]\right)^{\frac{1}{2}}}{\left|\mathcal{K}\left[\left\{P_{\mu}\right\} ; k\right]\right|}
$$

only if

$$
\forall P_{\mu^{\prime}}, P_{\mu^{\prime \prime}} \in\left\{P_{\mu}\right\} \forall n \in \mathbb{N}:\left\|\left[\left(U^{n} P_{\mu^{\prime}} U^{\dagger n}\right), P_{\mu^{\prime \prime}}\right]\right\|_{2}<2 d^{\frac{3}{2}} \sqrt{\epsilon},
$$

where $d=\operatorname{dim} \mathcal{H}$ and $\|\cdot\|_{2}$ denotes the Hilbert-Schmidt operator norm.

[1] Scherer, A., Soklakov, A. N., and Schack, R., Phys. Lett. A 326, 307 (2004). 
[2] Scherer, A., and Soklakov, A. N., e-rpint: quant-ph/0405080, (2004).

[3] Griffiths, R., J. Stat. Phys. 36, 219 (1984).

[4] Omnès, R., J. Stat. Phys. 53, 893, 933, 957 (1988).

[5] Gell-Mann, M., and Hartle, J. B., "Quantum Mechanics in the Light of Quantum Cosmology", in Complexity, Entropy, and the Physics of Information, edited by W. H. Zurek, Addison Wesley, Redwood City, CA, 1990, pp. 425-458.

[6] Dowker, H. F., and Halliwell, J. J., Phys. Rev. D 46, 1580 (1992).

[7] Gell-Mann, M., and Hartle, J. B., Phys. Rev. D 47, 3345 (1993).

[8] Hartle, J. B., in Proceedings of the 11th Nishinomiya-Yukawa Symposium, edited by K. Kikkawa, H. Kunitomo, and H. Ohtsubo, World Scientific, Singapore, 1998.

[9] Halliwell, J. J., Phys. Rev. D 58, 105015 (1998).

[10] Soklakov, A. and Schack, R., Phys. Rev. E 66, 036212 (2002).

[11] Poulin, D., Phys. Rev. A 65, 42319 (2002).

[12] Diosi, L., Phys. Rev. Lett. 92, 170401 (2004).

[13] Condition (4) is trivially a sufficient single-iteration decoherence condition both in the finegrained as well as in the coarse-grained case.

[14] In [6] a weaker condition was proposed, with $\left|\operatorname{Re} \mathcal{D}_{U, \rho}\left[h_{\vec{\alpha}}, h_{\vec{\beta}}\right]\right|$ instead of $\left|\mathcal{D}_{U, \rho}\left[h_{\vec{\alpha}}, h_{\vec{\beta}}\right]\right|$ on the left-hand side of the inequality (7). 